\title{
Desarrollo y evaluación de un método racional y no destructivo para la toma de muestras de maderas blandas utilizadas en análisis químicos
}

\section{Development and evaluation of a rational and nondestructive sampling methodology for softwoods used in chemical analyses}

\author{
Ricardo Musule!', Guadalupe M. Bárcenas-Pazos², M. del Rosario Pineda-López', Eric P. Houbron³ y \\ Lázaro R. Sánchez-Velásquez ${ }^{1 *}$
}

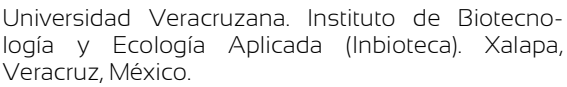

\author{
Instituto de Ecología A.C. Red Ambiente y Susten- \\ tabilidad. Xalapa, Veracruz, México. \\ Universidad Veracruzana. Facultad de Ciencias
}

Químicas, Orizaba, Veracruz, México.
* Autor de correspondencia. lasanchez@uv.mx

\begin{abstract}
RESUMEN
La madera ha sido un recurso natural ampliamente utilizado por la humanidad. Particularmente, la madera de coníferas destaca por su importancia ecológica, económica y distribución geográfica en comparación con otros taxa. Algunos usos de las diferentes especies maderables se basan en la evaluación de sus propiedades físicas, así como en el análisis de sus principales propiedades químicas. Para esto último, es deseable una metodología óptima para la toma de muestra de madera (TMM) en árboles en pie. Estas metodologías son escasas y pocas consideran anticipadamente en conjunto, tanto la cantidad necesaria de madera para las determinaciones químicas (toma de muestra racional) como también una toma de muestra no destructiva (sin talar los árboles). Por esta razón el objetivo del presente estudio fue desarrollar una metodología paso a paso que describa la TMM de coníferas de forma racional y no destructiva. La metodología TMM se evaluó en Abies religiosa a lo largo de diferentes sitios de muestreo y consiste en cuatro pasos: 1) Definir la cantidad de madera necesaria; 2) Determinar la densidad básica de la madera (parámetro más importante en la precisión de posteriores cálculos); 3) Calcular el volumen a extraer y 4) Tomar las muestras de madera en campo. Los resultados mostraron que la cantidad de madera de $A$. religiosa obtenida con la metodología fue mayor a la necesaria, sin presentar diferencias significativas $(P>0.05)$ entre los sitios de muestreo. En conclusión, la metodología fue exitosa para esta conífera, sin embargo, se requieren estudios posteriores para determinar si podrá aplicarse en otras especies de maderas de coníferas.
\end{abstract}

Palabras Clave: Abies religiosa, análisis químicos, árbol en pie, cantidad de biomasa, densidad de la madera, maderas de coníferas.

\begin{abstract}
Wood has been a natural resource widely used by mankind. Particularly, conifer wood is important because of its ecology, economy and geographic distribution in comparison with others taxa. Usage of different timber species is based on the evaluation of their physical properties and also on the analysis of their main chemical properties. Thus, in order to achieve good chemical determinations, it is required an optimal methodology for sample collection of wood (SCW) in standing trees. This kind of methodologies are scarce, moreover just a few of them consider ahead of time the amount of wood needed for chemical analysis (rational sample collection) and at that at the same time they are a nondestructive sample collection (without cutting down trees). For that reason, the aim of this study was to develop a new methodology which describe SCW of conifers in a rational and nondestructive way. This SCW methodology was evaluated in Abies religiosa along of different sampling sites and consists of four steps: 1) To define the amount of wood needed; 2) To determine the basic density of wood (main parameter in the precision of subsequent calculations); 3 ) To estimate the volume to extract and 4) To take samples of wood in field. Our results showed that with this methodology we obtained greater amount of $A$. religiosa wood than the needed without showing significant differences $(P>0.05)$ between sampling sites. In conclusion, this methodology was successful for this conifer, however future studies are required in order to determine if it can be applied in other softwood species.
\end{abstract}

KEYWORDS: Abies religiosa, chemical analysis, standing trees, biomass quantity, wood density, conifer wood. 


\section{INTRODUCCIÓN}

La madera es un recurso forestal polimérico, con características heterogéneas y anisotrópicas, que se forma durante el crecimiento de plantas leñosas (Zhu, Shi, Fang, Liu y Ji, 2015). En la actualidad el uso de la madera se considera clave para la mitigación del cambio climático debido a que favorece la captura y almacenamiento del carbono atmosférico y puede reducir emisiones a través de sus diversos usos (Matsumoto, Oka, Mitsuda, Hashimoto, Kayo, Tsunetsugu y Tonosaki, 2016). Se espera un aumento en la demanda de productos maderables y consecuentemente un interés en la incorporación de prácticas sustentables e intensivas en el manejo forestal enfocado a una mayor calidad de productos maderables (Hevia, ÁlvarezGonzález y Majada, 2016). Los bosques de coníferas se encuentran entre los ecosistemas más trascendentes de nuestro planeta (Smith y Hinckley, 1995), son una fuente importante de madera para la industria forestal y actualmente en México representan los principales recursos de abastecimiento maderable (Martínez-Antúnez, Hernández-Díaz, Wehenkel y LópezSánchez, 2015).

Los usos eficientes de las maderas se determinan mediante la exploración y evaluación de sus propiedades físicas y mecánicas, que a su vez también proporcionan información valiosa para entender la estructura y función de los ecosistemas terrestres, así como para predecir sus respuestas al cambio climático global (Zhu et al., 2015). Otro criterio fundamental para determinar el uso de las maderas, se relaciona con la caracterización de sus propiedades químicas, debido a que estas afectan directamente a los procesos industriales que las usan como materia prima (Farmer, 1967). Para la realización de cualquier análisis físico, mecánico o químico, entre otros, es necesaria la recolección de muestras de madera en diversas cantidades, incluyendo el derribo en múltiples ocasiones de árboles completos (métodos destructivos).

La toma de muestras es esencial para la caracterización de la madera y se relaciona con la selección óptima del tamaño de la muestra y de la unidad de muestreo, así como de los puntos de medición (extracción o distribución espacial), entre otros. Por lo tanto, un muestreo inapropiado puede causar errores de medición y tener importantes efectos en los resultados (Sandak, Sandak, y Riggio, 2015). En la actualidad, pocos procedimientos describen sistemáticamente una metodología no destructiva (evitando derribar el árbol) y de bajo costo en la toma de muestra de madera en campo (Bauer, 1975; ISO 3129, 1975). La gran mayoría de las metodologías descritas en la literatura, que cumplen estos requisitos, tienen como objetivo realizar análisis dendrocronológicos (Chave, 2006; Steenkamp, Van Rooyen y Van Rooyen, 1999; Wiemann y Williamson, 2013; Williams, Gagen, Walsh y Bidin, 2015). Sin embargo, estas metodologías no contemplan ni describen la toma racional de muestras de madera (cantidad necesaria de biomasa por extraer) con base en análisis químicos, por ejemplo, análisis de polímeros estructurales, poder calorífico, contenido mineral, entre otros. Consecuentemente, el desarrollo de una metodología de toma de muestra de maderas, racional y no destructiva sería de gran utilidad para realizar investigaciones sobre las propiedades químicas de la madera de coníferas.

\section{OBJETIVOS}

1) Desarrollar una metodología de toma de muestras de madera de coníferas en pie, no destructiva (minimizando el daño a los árboles muestreados) y que permita obtener la cantidad necesaria de material (racional) para utilizarse en análisis químicos.

2) Evaluar la metodología en bosques de Abies religiosa en diferentes sitios de muestreo.

\section{MATERIALES Y MÉTODOS}

\section{Modelo conceptual del método}

El modelo incluye cuatro pasos fundamentales para la toma de muestras de madera de coníferas en pie: 1) Definir la cantidad de madera a utilizar para los análisis químicos, 2) Determinar la densidad básica de la madera de la especie de interés, 3) Determinar el volumen de madera que se extraerá y 4) Tomar las muestras de madera en campo (Fig. 1).

\section{1) Definir la cantidad de madera a utilizar para los análisis químicos}

En este primer paso, es necesario responder a la pregunta ¿Cuánta biomasa (gramos) de madera se requiere para el estudio? Esto puede depender de múltiples factores, por ejemplo: el número y 
atributos de los árboles a muestrear, las especificaciones de los procedimientos analíticos físico-químicos a realizarse, la precisión del analista y de los métodos de análisis, entre otros. Por lo tanto, habrá que definir lo siguiente:

\section{1) Número y características de los árboles en el diseño} experimental. El diseño experimental y la obtención de un tamaño de muestra adecuado son cruciales para cualquier estudio de carácter científico (Clewer y Scarisbrick, 2001), por lo tanto, en este punto es necesario definir las unidades de estudio. Por ejemplo, las unidades de muestreo pueden corresponder a árboles $\left(U_{m}\right)$ de los cuales se extraerá la madera. Sin embargo, dependiendo del diseño experimental y su correspondiente tamaño de muestra se puede requerir el muestreo de árboles independientes $\left(U_{m}=1\right)$, o de un conjunto de árboles (muestras compuestas, $U_{m}=\mathrm{X}$ ) con determinadas características, como puede ser: edad, estado de salud, algún tipo de estrato/estado de desarrollo o zona geográfica, entre otros.

Por lo tanto, como punto de partida se requiere conocer y definir si se trabajará con árboles independientes o con un conjunto de árboles (definir cuantos árboles conformarán las muestras compuestas) ya que esto determinará la cantidad de madera $(\mathrm{g})$ requerida para los análisis químicos programados, e influirá directamente en las estimaciones de la cantidad de madera a extraer de los árboles en pie.

1.2) Número de análisis químicos. Entre los análisis químicos que se llevan a cabo rutinariamente para caracterizar una cierta madera se encuentra, por ejemplo, la determinación de los porcentajes de polímeros estructurales de la pared celular (celulosa, hemicelulosa y lignina), de material mineral (cenizas), de contenido de extractos y de proteínas, entre otros. Con base en los requerimientos de cada análisis se puede estimar la cantidad total de madera requerida (Tabla 1). Esto implica la definición del número de análisis que se requieren en un estudio en particular para estimar la cantidad de madera necesaria $\left(M_{a}\right)$, es decir la sumatoria de las cantidades de madera del conjunto de análisis a realizarse. Lo anterior se expresa en la ecuación 1 , donde $m_{1}$ corresponde a la cantidad de madera de un análisis determinado, $m_{2}$ a la cantidad de un segundo análisis, hasta llegar a $n$ número de análisis, lo cual dependerá de los objetivos del estudio en particular.

$$
M_{\alpha}=\sum\left(m_{1}+m_{2}+\ldots .+m_{n}\right)
$$

TABLA 1. Definir el número de análisis químicos a realizar y sus respectivas características.

\begin{tabular}{|c|c|c|c|}
\hline $\begin{array}{l}\text { Análisis } \\
\text { químico }\end{array}$ & $\begin{array}{l}\text { Adecuación de } \\
\text { las muestras } \\
\text { (pre- } \\
\text { procesamiento) }\end{array}$ & $\begin{array}{l}\text { Réplicas } \\
\text { analíticas }\end{array}$ & $\begin{array}{l}\text { Cantidad } \\
\text { de muestra } \\
\text { por } \\
\text { medición }\end{array}$ \\
\hline $\begin{array}{l}\text { Análisis } 1 \\
\text { Análisis } 2 \\
\text { Análisis n }\end{array}$ & $\begin{array}{l}\text { Sin procesar, } \\
\text { secado, molido, } \\
\text { tamizado, entre } \\
\text { otros. }\end{array}$ & $\begin{array}{l}\text { Sin réplica, } \\
\text { duplicado, } \\
\text { triplicado, } \\
\text { etc. }\end{array}$ & $\begin{array}{l}\text { Cantidad } \\
\text { en gramos } \\
\text { utilizados } \\
\text { por } \\
\text { medición }\end{array}$ \\
\hline
\end{tabular}

1.3) Factor de ajuste. De la cantidad de madera calculada en el paso anterior, es recomendable obtener una cantidad extra, previendo la posibilidad de que lleguen a ocurrir errores al efectuar los análisis, por ejemplo, la aparición de errores técnicos de los equipos o del analista. También es recomendable tener una reserva de material por si fuera necesario realizar un análisis no contemplado en la metodología inicial; o por la pérdida de muestra, debido a los diferentes pre-procesamientos y preparaciones de la madera para análisis como son los pretratamientos de secado, molido y tamizado.

Es recomendable usar un factor de ajuste $\left(F_{a}\right)$, por ejemplo, $F_{a}=0.25$, es decir considerar obtener un remanente del $25 \%$ del material por usar en el total de los análisis para casos imprevistos, este factor, al ser un parámetro sugerido, puede ser replanteado y modificado con base en las necesidades de cada estudio en particular.

\section{4) Cálculo de la cantidad de madera total requerida.}

Habiendo seguido los pasos anteriores se procede a calcular la cantidad de madera total requerida $\left(M_{T}\right)$ por árbol o árboles (unidad de muestreo), la cual puede ser obtenida utilizando la ecuación 2.

$$
M_{T}=\frac{M_{\alpha}+\left(M_{\alpha} \times F_{a}\right)}{U_{m}}
$$




\section{2) Determinar la densidad básica de la madera}

El valor de la densidad básica $(\mathrm{Db})$ de cualquier madera en estudio, puede ser obtenida de la bibliografía, o calculándola experimentalmente mediante datos de campo.

2.1) Determinación de la $\mathrm{Db}$ con revisión de bibliografia. Para esta primera opción, con la revisión bibliográfica del parámetro $\mathrm{Db}$ de la madera se puede encontrar el valor para la especie de interés específicamente, o en su defecto para una especie semejante, por ejemplo por género. $\mathrm{La} \mathrm{Db}$ de las maderas puede variar debido al efecto de múltiples factores (Wiemann y Williamson, 2013). Este parámetro es propio de cada especie vegetal y se asocia, entre otros factores, con las condiciones climáticas donde los individuos crecieron (Ordóñez Díaz, Galicia, Venegas, Hernández, Ordóñez Díaz, Dávalos-Sotelo, 2015; Goche-Télles, Velázquez-Martínez, Borja-de la Rosa, CapulínGrande y Palacios-Mendoza., 2011). Cabe señalar que al seleccionar este paso para determinar la $\mathrm{Db}$ se puede encontrar un amplio rango de valores reportados para este parámetro en una misma especie, lo cual dependerá de la cantidad y confiabilidad de las fuentes de información. Con base en lo anterior, se podría esperar una mayor incertidumbre de las estimaciones al utilizar este paso. Entre la basta información bibliográfica acerca de la $\mathrm{Db}$ de maderas, Ordóñez Díaz et al. (2015) hacen una recopilación del parámetro para una gran cantidad de maderas mexicanas.

2.2) Cálculo de la Db del árbol en pie. La segunda opción, es decir para la determinación del parámetro Db del árbol en pie, sugerimos primero un muestreo piloto en campo y estimar así una densidad básica promedio de la madera $\left(D_{B}\right)$. Existen diferentes métodos descritos en la literatura para calcular la $\mathrm{Db}$ del árbol en pie: el método empírico, el método de máximo contenido de humedad y el método de desplazamiento de agua (Manzo y Hernández, 1997; Wiemann y Williamson, 2013).

\section{3) Determinar el volumen de madera que se extraerá}

Utilizando los parámetros masa total de madera requerida $\left(M_{T}\right)$ y la densidad básica de la madera promedio $\left(D_{B}\right)$ que se obtuvo de la bibliografía o del árbol en pie o (punto 2.1 o 2.2), es posible estimar el volumen de extracción $\left(V_{E}\right)$ en $\mathrm{cm}^{3}$ (ecuación 3), que se requiere para análisis químicos.

$$
V_{E}=\frac{M_{T}}{D_{B}}
$$

\section{4) Tomar las muestras de madera en campo}

Una vez que se ha estimado el volumen requerido, como se describe en el paso anterior, se seleccionan las herramientas y equipos con las que se extraerá la madera en campo. Para este estudio se utilizó un berbiquí (taladro manual), con capacidad para usar diferentes tamaños y tipos de brocas. La selección de la broca y sus respectivas dimensiones se describen a continuación: 4.1) Selección de la broca a utilizar. En el mercado existen diferentes tipos y tamaños de brocas comerciales, con los que se puede realizar la extracción manual de la madera. Utilizado las dimensiones diámetro $(D)$ y longitud $(L)$ de determinada broca, se puede estimar el volumen de la misma $\left(V_{B}\right)$ y por ende el volumen de madera que se puede obtener al utilizar determinada broca para la extracción (ecuación 4).

$$
V_{B}=\left(\frac{\pi+D^{2}}{4}\right) \times L
$$

Posteriormente, se debe verificar que el volumen de la broca sea igual o poco mayor al requerido para obtener la masa necesaria en los análisis $\left(M_{T}\right)$. Para esto último debe cumplirse que $V_{E} \leq V_{B}$ y con ello se esperaría que la broca seleccionada sea adecuada. Adicionalmente, una vez seleccionada la broca hay que extraer el volumen necesario de madera $\left(V_{E}\right)$, por lo que se requiere definir una longitud de penetración de la broca $\left(L_{P}\right)$, lo cual se puede hacer al despejar la ecuación 4:

$$
L_{p}=\left(\frac{V_{B}(4)}{\pi \times D^{2}}\right)
$$

En caso de no encontrar un tamaño de broca comercial que cumpla con el supuesto $\left(V_{E} \leq V_{B}\right)$, o en su defecto por ya contar con un cierto tamaño de broca, se pueden presentar los siguientes problemas: el primero es que $V_{B}$ puede ser demasiado grande, la opción a esta situación se soluciona al definir una cierta longitud de penetración $\left(L_{P}\right)$ usando la ecuación 5 , modificando así la profundidad de extracción hasta lograr la adecuada. Una segunda situación podría ser que $V_{B}$ sea insuficiente; en este caso se requerirá hacer extracciones múltiples ( $N$ es el número de extracciones) del volumen de la broca $\left(V_{B N}\right)$, hasta cumplir con $V_{E} \leq V_{B N}$ (ecuación 6).

$$
V_{B N}=\frac{\pi \times D^{2}}{4} \times L \times N
$$


4.2) Extracción de las muestras de madera en campo. Una vez que se tiene el berbiquí y la broca seleccionada y establecido el procedimiento de extracción, los pasos son los siguientes: Se sugiere que la altura y la orientación a las que se realizará la extracción sean, a la altura de diámetro normal (DN), es decir a $1.3 \mathrm{~m}$ de la base del fuste y con la perforación en la orientación opuesta a la pendiente. Los orificios que resultarán de la extracción deben ser sellados con cera o madera para evitar daños fitosanitarios a los árboles muestreados. Las muestras de madera deben ser cuidadosamente colectadas, evitando contaminación por corteza u otro tipo de material y almacenadas en recipientes adecuados (bolsas u otro tipo de recipiente limpio) para su traslado y posterior análisis en laboratorio (Fig. 1).

\section{Validación de la metodología}

Caso práctico ilustrativo en madera de Abies religiosa

Para validar la metodología propuesta se describe el siguiente caso práctico aplicado a seis árboles de $A$. religiosa $(n=6)$ que crecieron en seis diferentes sitios a lo largo de un gradiente altitudinal (36 árboles en total). Se comprobó mediante un ANOVA de un factor (donde la variable respuesta fue la cantidad de madera obtenida por el método desarrollado y el factor los diferentes sitios de muestreo, utilizando transformación a rangos de la variable respuesta (Conover e Iman, 1981)) que la cantidad de madera obtenida por cada árbol no cambió significativamente entre de los diferentes sitios de muestreo.

La madera fue extraída para realizar los siguientes análisis químicos: porcentaje de polímeros estructurales (Richardson, 2004), porcentaje de cenizas (Sluiter, Hames, Ruiz, Scarlata, Suiter y Templenton, 2008) y determinación de poder calorífico superior (Yang, Kim, Sagong y Han, 2016). Además se verificó que la cantidad de muestra obtenida en campo fuera mayor a la cantidad necesaria para los análisis químicos $\left(M_{a}\right)$.

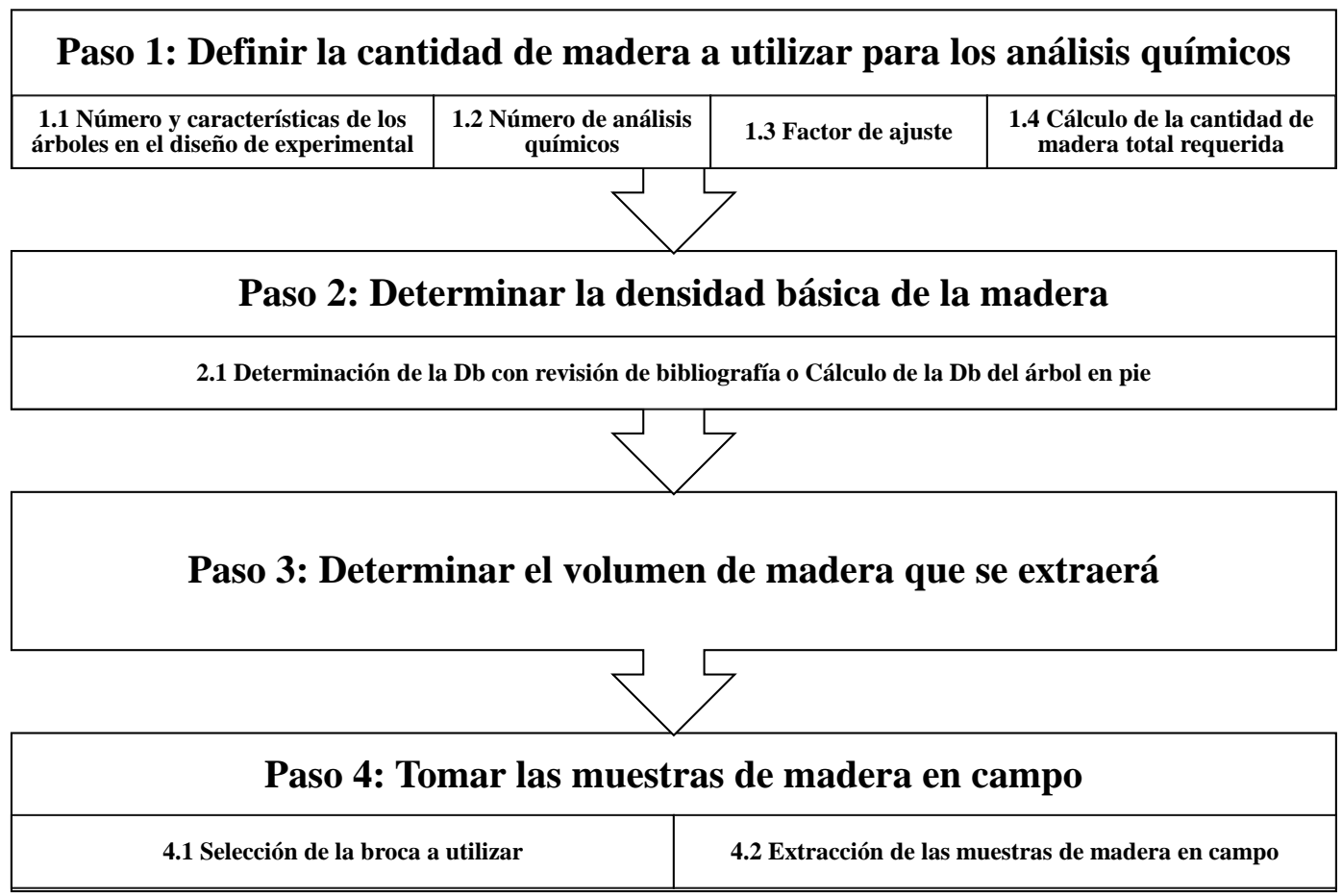

FIGURA 1. Diagrama de flujo de la metodología de toma de muestras de madera en árboles en pie. 


\section{RESULTADOS}

\section{Diagrama del modelo de la metodología}

La metodología de toma de muestras de madera se ilustra en el diagrama de flujo de la figura 1 para facilitar su uso.

\section{Validación de la metodología}

La metodología anteriormente descrita se validó para el caso práctico ilustrativo en la toma de muestras de madera de $A$. religiosa siguiendo los pasos:

1) Definir la cantidad de madera a utilizar para análisis químicos

\section{1) Número y características de los árboles en el diseño de} experimental. Se definió como unidad de muestreo a individuos (árboles) de $A$. religiosa, es decir, $U_{m}=1$.

1.2) Número de análisis químicos. En la tabla 2 se presenta un ejemplo para la extracción de la madera necesaria para tres tipos de análisis químico. Una vez que se definió el tipo y número de análisis a realizar y sus respectivos atributos, con la ecuación 1 se calculó la cantidad de madera requerida para los análisis $(M a)$; en este caso particular fue de $10.5 \mathrm{~g}$.

1.3 Factor de ajuste. Tomando en consideración que durante el procesamiento y análisis de la madera pueden ocurrir perdidas de muestra aunado a una posible aparición de errores imprevistos, se seleccionó un factor de ajuste $\left(F_{a}\right)$ del $25 \%$, es decir, $F_{a}=0.25$.

TABLA 2. Estimación de la cantidad de madera para análisis químicos en la madera de $A$. religiosa.

\begin{tabular}{lllll}
\hline Análisis químico & Pre-procesamiento & $\begin{array}{l}\text { Réplicas } \\
\text { analíticas }\end{array}$ & $\begin{array}{l}\text { Procedimiento y } \\
\text { cantidad de muestra }\end{array}$ & $\begin{array}{l}\text { Total de muestra } \\
\text { necesaria por árbol }(\mathrm{Ma})\end{array}$ \\
\hline $\begin{array}{l}\text { Polímeros estructurales } \\
\text { (Richardson, 2004) }\end{array}$ & $\begin{array}{l}\text { Secado, molido y } \\
\text { tamizado }\end{array}$ & Triplicado & $0.5 \mathrm{~g}(\mathrm{BS})$ & $\begin{array}{l}(\mathrm{O} .5 \times 3)= \\
1.5 \mathrm{~g}\left(\mathrm{~m}_{1}\right)\end{array}$ \\
\hline $\begin{array}{l}\text { Cenizas (Sluiter et al., } \\
\text { 2008) }\end{array}$ & $\begin{array}{l}\text { Secado, molido y } \\
\text { tamizado }\end{array}$ & Triplicado & $2 \mathrm{~g}(\mathrm{BS})$ & $(2 \times 3)=$ \\
\hline $\begin{array}{l}\text { Poder calorífico superior } \\
\text { (Yang et al., 2016) }\end{array}$ & $\begin{array}{l}\text { Secado, molido, } \\
\text { tamizado y }\end{array}$ & Triplicado & $1 \mathrm{~g}(\mathrm{BS})$ & $(1 \times 3)=$ \\
& pellet & & & $3 \mathrm{~g}\left(\mathrm{~m}_{3}\right)$ \\
\hline Ec.l $M a=m_{1}+m_{2}+m_{3}=$ & $1.5 \mathrm{~g}+6 \mathrm{~g}+3 \mathrm{~g}=$ & & $10.5 \mathrm{~g}$ \\
\hline
\end{tabular}

(BS)=Base seca, madera libre de humedad

\subsection{Cálculo de la cantidad de madera total requerida. Se} calculó la masa total $\left(M_{T}\right)$ a extraer utilizando los parámetros definidos en los puntos anteriores $U_{m}, M_{a} y F_{a}$ :

$$
M_{T}=\frac{M_{\alpha}+\left(M_{\alpha} \times F_{a}\right)}{U_{m}}=\frac{10.5+(10.5 \times 0.25)}{1}=13.1 \mathrm{~g}
$$

\section{2) Determinar la densidad básica de la madera}

Se calculó la Db de la madera en campo, es decir, siguiendo la segunda opción planteada por la metodología propuesta, se realizó un muestreo piloto en campo, en el que aleatoriamente se seleccionaron 10 árboles (de los 36 del estudio) y se les determinó su Db mediante el método empírico, mesurando las dimensiones de las virutas de madera obtenidas con un taladro Pressler (Haglöf Sweden, $5 \mathrm{~mm}$ de diámetro). Se encontró que la densidad básica promedio $\left(D_{B}\right)$ de $A$. religiosa fue de $0.32 \mathrm{~g} / \mathrm{cm}^{3} \pm 0.4 \mathrm{~g} / \mathrm{cm}^{3}$.

\section{3) Determinar el volumen de madera que se extraerá}

Utilizando los parámetros calculados anteriormente $M_{T}$ у $D_{B}$ y sustituyéndolos en la ecuación 3 , se determinó el volumen que se requiere extraer de madera $\left(V_{E}\right)$ de $A$. religiosa, el cual fue de 40.9 $\mathrm{cm}^{3}$.

$$
V_{E}=\frac{M_{T}}{D_{B}}=\frac{13.1}{0.32}=40.9 \mathrm{~cm}^{3}
$$




\section{4) Tomar las muestras de madera en campo}

4.1) Selección de la broca a utilizar. Se seleccionó una broca tipo gusano de 1 pulgada de diámetro (marca Surtek), la cual tiene una longitud de extracción de hasta de $11 \mathrm{~cm}$. Por lo que su volumen de broca $\left(V_{B}\right)$ es de hasta $55.7 \mathrm{~cm}^{3}$, valor que se estimó de la siguiente manera.

$$
V_{B}=\frac{\pi \times D^{2}}{4} \times L=\frac{\pi \times(2.54)^{2}}{4} \times 11=55.7 \mathrm{~cm}^{3}
$$

Por lo que se cumple $V_{E} \leq V_{B}$; de manera que la broca seleccionada es adecuada para la extracción en campo. Adicionalmente, se definió la longitud de extracción $\left(L_{P}\right)$ apropiada para obtener $V_{E}=40.9 \mathrm{~cm}^{3}$, utilizando la ecuación 5 :

$$
L_{p}=\left(\frac{V_{B}(4)}{\pi \times D^{2}}\right)=\frac{40.9(4)}{\pi(2.54)^{2}}=8 \mathrm{~cm}
$$

La longitud de penetración de la broca fue de $8 \mathrm{~cm}$ de profundidad para obtener los parámetros volumen $\left(V_{E}\right)$ y masa $\left(M_{T}\right)$ necesaria de madera.

\section{2) Extracción de las muestras de madera en campo.}

Utilizando un berbiquí (marca Surtek) equipado con la broca seleccionada (Fig. 2), se extrajeron virutas de madera de forma manual a una profundidad de $8 \mathrm{~cm}$, aproximadamente al DN en cada árbol, evitando la corteza de los árboles y colectando sólo tejido xilemático el cual fue guardado en bolsas de plástico inmediatamente después de su recolección, que fueron selladas para evitar la contaminación de las muestras. Los orificios de la extracción se sellaron con taquetes de madera para evitar daños a los árboles. Las muestras fueron transportadas al laboratorio para determinar las cantidades de madera obtenidas por la metodología de toma de muestra.

En laboratorio se cuantificó la cantidad de madera de $A$. religiosa obtenida por cada árbol (36 árboles) a lo largo de los seis sitios de muestreo aplicando la metodología definida para la toma de muestra. En promedio se obtuvieron $14.67 \mathrm{~g} \pm 2.0 \mathrm{~g}$ de madera y no se encontraron cambios significativos entre la cantidad de madera obtenida a lo largo de los diferentes sitios de muestreo $\left(F_{5,30}=1.109 P=0.377\right.$, Fig. 3).
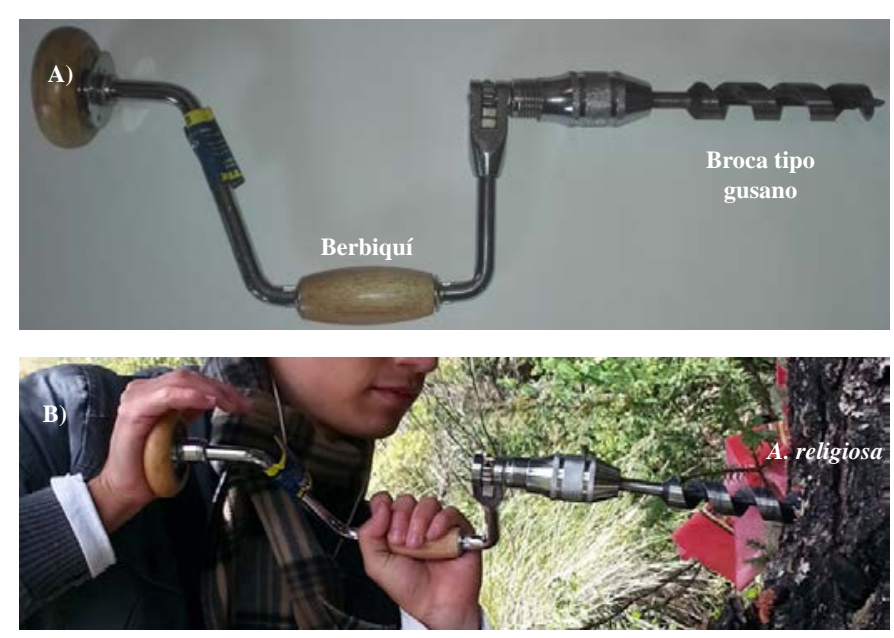

Figura 2. Equipo de extracción de madera (A) y toma de muestras de madera de $A$. religiosa en campo (B).

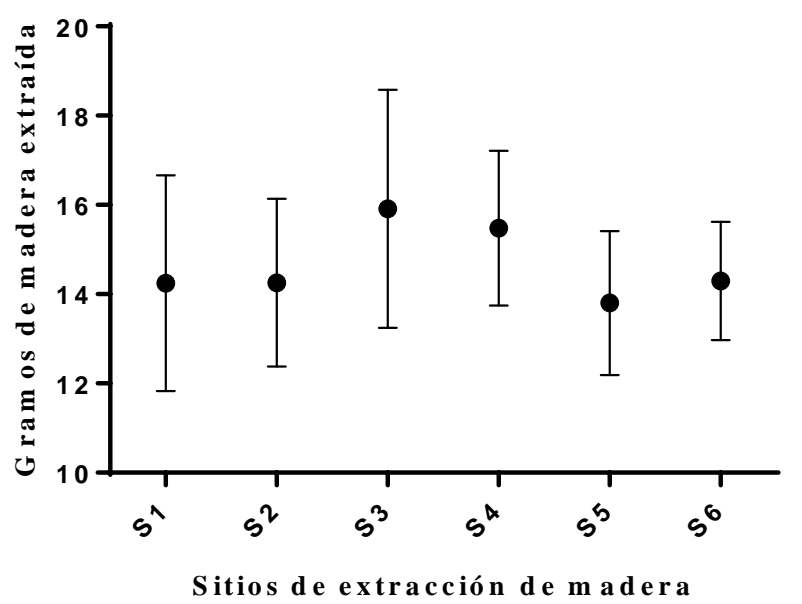

FIgURA 3. Cantidad de madera (g) extraída de árboles en pie de A. religiosa en seis sitios de estudio.

\section{DISCUSIÓN}

Se desarrolló una metodología de toma de muestra de maderas para árboles en pie y se validó en $A$. religiosa. La metodología consta de cuatro pasos con los que se obtiene una cantidad necesaria de muestra para realizar análisis químicos. $\mathrm{Al}$ aplicar la metodología y sus respectivos pasos se evita la tala de los árboles muestreados y también se amplían las fronteras de estudio con preguntas articuladas entre las propiedades físico-químicas de la madera y la ecología. De igual manera es útil en áreas naturales protegidas donde no se permite realizar la tala de árboles y el desarrollo de investigación científica es relevante (DávalosSotelo, 2016). 
La metodología comprende como paso inicial definir una cantidad necesaria de muestra, lo que le da la característica de ser racional en contraste con otras metodologías de toma de muestra no destructivas (Bauer, 1975; Steenkamp et al., 1999; Wiemann y Williamson, 2013; Williams et al., 2015), en las que únicamente se indica cómo realizar la extracción de la madera sin previamente definir la cantidad que se necesita.

En el segundo paso, se utiliza la $\mathrm{Db}$, un parámetro sumamente estudiado y que a su vez se encuentra ampliamente documentado en la literatura para muchas especies de maderas (Ordóñez Díaz et al., 2015). El uso de la Db para la estimación de biomasa es una técnica ampliamente practicada (Wassenberg, Chiu, Guo y Spiecker, 2015; Wiemann y Williamson, 2014; Wiemann y Williamson, 2013). Al conocer cuantitativamente la $\mathrm{Db}$ se facilita y se permite realizar los pasos siguientes de una manera fácil y confiable. Con este método es sencillo calcular el volumen de madera que se requiere extraer.

Por último, para la toma de muestras de madera en campo a través de un taladro manual (berbiquî) y una broca (estas herramientas son accesibles y económicas, aunado a que no requieren de uso de electricidad), lo que les confiere una versatilidad en el uso de las mismas en campo, en comparación con técnicas que utilizan dispositivos eléctricos (Bauer, 1975).

\section{Validación de la metodología}

Se probó que la metodología de toma de muestra es efectiva para madera de $A$. religiosa, debido a que se obtuvo en todos los casos una biomasa de muestreo un poco mayor a la requerida en los análisis $\left(M_{a}\right)$, es decir, $14.67 \mathrm{~g} \pm 2.0 \mathrm{~g}>10.5 \mathrm{~g}$ en todos los casos. De esta manera se obtuvo una cantidad suficiente de madera para suplir la demanda de los posteriores análisis químicos. Sin embargo, en pocos casos la cantidad de madera obtenida no fue mayor al $M_{T}$ calculado (13.1 g) pero fue suficiente. Lo anterior sugiere que estas ligeras diferencias se debieron a la variación intrínseca de la Db de la madera de $A$. religiosa, es decir, se explica por la determinación en campo de la $\mathrm{Db}$ promedio $\left(D_{B}\right)$ para madera de $A$. religiosa la cual fue de $0.32 \mathrm{~g} / \mathrm{cm}^{3} \pm 0.4 \mathrm{~g} / \mathrm{cm}^{3}$, valor con el que se realizaron varios cálculos de la metodología de toma de muestra. La amplia variación de la $\mathrm{Db}$ se ha publicado en la literatura para $A$. religiosa, con valores que van desde un mínimo de $0.14 \mathrm{~g}$ hasta un máximo de $0.59 \mathrm{~g} / \mathrm{cm}^{3}$ (Goche-Télles et al., 2000), con densidades promedios de $0.36 \mathrm{~g} / \mathrm{cm}^{3}-0.38 \mathrm{~g} / \mathrm{cm}^{3}$ (Ordóñez Díaz et al., 2015; Goche-Télles et al., 2000). Se puede observar que los datos determinados en campo para la $\mathrm{Db}$ de $A$. religiosa se encuentran por debajo de la densidad promedio encontrada en la literatura y en caso de haber optado por utilizar las densidades promedio de la literatura en lugar de la determinación en campo el volumen de extracción calculado hubiera sido menor, al igual que la longitud de penetración $\left(L_{P}\right)$ y probablemente se hubieran obtenido menores cantidades de madera, por lo que corregir la extracción de madera por el factor $F_{a}=0.25$ resultó apropiado.

Con base en lo anterior, se puede esperar que en especies maderables con amplia variabilidad de $\mathrm{Db}$ se obtendrá una menor precisión de los cálculos al aplicar la metodología de toma de muestra, por lo cual es recomendable usar factores de ajuste altos $\left(F_{a}\right)$ en estos casos. Por otro lado, en especies forestales con $\mathrm{Db}$ con menor variación se espera mayor precisión en la toma de muestra siguiendo la metodología propuesta en este estudio. Por lo anterior, es necesario y recomendable evaluar la metodología de toma de muestra de madera en otras especies de coníferas y especies con madera blanda.

\section{CONCLUSIONES}

Se desarrolló una metodología novedosa de toma de muestras de maderas en árboles en pie paso a paso, con la cual se puede obtener una cantidad de madera para análisis químicos de manera racional, no destructiva y de bajo costo. Adicionalmente, la metodología es accesible y reproducible, lo que se validó mediante la toma de muestras de madera de $A$. religiosa a lo largo de diferentes sitios de muestreo, no encontrándose cambios significativos entre ellos. Se observó que la metodología fue efectiva para obtener la cantidad necesaria de madera calculada para los posteriores análisis químicos. La precisión de los cálculos al aplicar la metodología de toma de muestras de madera, estará muy relacionada con la Db debido a que este parámetro es crucial en los cálculos. Cabe señalar que esta metodología podría ser útil para ser aplicada en otras especies de árboles con madera blanda, por lo que son necesarios más estudios. 


\section{RECONOCIMIENTOS}

Se agradece a Rogelio Lara González y a Yitzendi López Serrano por su valioso apoyo en el trabajo de campo. Finalmente, Ricardo Musule Lagunes agradece al Consejo Nacional de Ciencia y Tecnología (Conacyt) por la beca de posgrado otorgada (344898)

\section{REFERENCIAS}

Bauer, E. (1975). Method for removing wood samples from trees. Forest Science, 21(1), 51-52.

Chave, J. (2006). Medición de densidad de madera en árboles tropicales manual de campo. France: Sixth Framework Programme Pan-Amazonia7.

Clewer, A. G., \& Scarisbrick, D. H. (2001). Practical statistics and experimental design for plant and crop science. Inglaterra: John Wiley \& Sons.

Conover, W. J. e Iman, R. L. (1981). Rank transformations as a bridge between parametric and nonparametric statistics. The American Statistician, 35(3), 124-129.

Dávalos-Sotelo, R. (2016). El papel de la investigación científica en la creación de las áreas naturales protegidas. Madera y Bosques, 22(1), 7-13. doi: 10.21829/myb.2016.221474

Farmer, R. H. (1967). Chemistry in the Utilization of Wood. Inglaterra: Pergamon Press.

Goche-Télles, J. R., Fuentes-Salinas, M., Borja-de la Rosa, A., \& Ramírez-Maldonado, H. (2000). Variación de las propiedades físicas de la madera en un árbol de Abies religiosa y de Pinus ayacahuite var. veitchii. Revista Chapingo Serie Ciencias Forestales y del Ambiente, 6(1), 83-92.

Goche-Télles, J. R., Velázquez-Martínez, A., Borja-de la Rosa, A., Capulín-Grande, J., \& Palacios-Mendoza, C. (2011). Variación radial de la densidad básica en Pinus patula Schldl et Cham. de tres localidades en Hidalgo. Revista Mexicana de Ciencias Forestales, 2(7), 71-78.

Hevia, A., Álvarez-González, J. G., \& Majada, J. (2016). Comparison of pruning effects on tree growth, productivity and dominance of two major timber conifer species. Forest Ecology and Management, 374, 82-92. doi: 10.1016/j.foreco.2016.05.001

ISO 3129. (1975). Wood-Sampling Methods and General Requirements for Physical and Mechanical Tests. Suiza: International Organization for Standardization.

Manzo, S. V., \& Hernández, J. V. (1997). Método empírico para estimar la densidad básica en muestras pequeñas de madera. Madera y Bosques, 3(1), 81-87. doi: 10.21829/myb.1997.311381
Martínez-Antúnez, P., Hernández-Díaz, J. C., Wehenkel, C., \& López-Sánchez, C. A. (2015). Estimación de la densidad de especies de coníferas a partir de variables ambientales. Madera y Bosques, 21(1), 23-33. doi: 10.21829/myb.2015.211430

Matsumoto, M., Oka, H., Mitsuda, Y., Hashimoto, S., Kayo, C., Tsunetsugu, Y., \& Tonosaki, M. (2016). Potential contributions of forestry and wood use to climate change mitigation in Japan. Journal of Forest Research, 21(5), 211222. doi: 10.1007/s10310-016-0527-4

Ordóñez Díaz, J. A. B., Galicia Naranjo, A., Venegas Mancera, N. J., Hernández Tejeda, T., Ordóñez Díaz, M. de J., \& Dávalos-Sotelo, R. (2015). Densidad de las maderas mexicanas por tipo de vegetación con base en la clasificación de J. Rzedowski: compilación. Madera y Bosques, 21(Número especial), 77-126. doi: 10.21829/myb.2015.210428

Richardson, A. D. (2004). Foliar chemistry of balsam fir and red spruce in relation to elevation and the canopy light gradient in the mountains of the northeastern United States. Plant and Soil, 260(1-2), 291-299. doi: 10.1023/B:PLSO.0000030179.02819.85

Sandak, A., Sandak, J., \& Riggio, M. (2015). Estimation of physical and mechanical properties of timber members in service by means of infrared spectroscopy. Construction and Building Materials, 101, 1197-1205. doi: 10.1016/j.conbuildmat.2015.06.063

Sluiter, A., Hames, B., Ruiz, R., Scarlata, C., Sluiter, J., \& Templeton, D. (2008). Determination of ash in biomass. EUA: Laboratory Analytical Procedure (LAP). Nrel/Tp510-42622.

Smith, W. K., \& Hinckley, T. M. (1995). Resource physiology of conifers: acquisition, allocation, and utilization. EUA: Academic Press.

Steenkamp, C. J., van Rooyen, M. W., \& Van Rooyen, N. (1999). A non-destructive sampling method for dendrochronology in hardwood species. Southern African Forestry Journal, 186(1), 5-7. doi: 10.1080/10295925.1999.9631235

Wassenberg, M., Chiu, H. S., Guo, W., \& Spiecker, H. (2015). Analysis of wood density profiles of tree stems: incorporating vertical variations to optimize wood sampling strategies for density and biomass estimations. Trees - Structure and Function, 29(2), 551-561. doi: 10.1007/s00468-014-1134-7

Wiemann, M., \& Williamson, G. B. (2013). Biomass Determination Using Wood Specific Gravity from Increment Cores. EUA: USDA Forest Service FPL-GTR225

Wiemann, M. C., \& Williamson, G. B. (2014). Wood specific gravity variation with height and its implications for biomass estimation. EUA: USDA Forest Service FPL-RP677. 
Williams, R. E., Gagen, M. H., Walsh, R. P. D., \& Bidin, K. (2015). On the development of a drill-borer for sampling tropical supra-hardwoods: An example using the Borneo Ironwood Eusideroxylon zwageri. Dendrochronologia, 35, 99-104. doi: 10.1016/j.dendro.2015.07.004

Yang, I., Kim, S., Sagong, M., \& Han, G.-S. (2016). Fuel characteristics of agropellets fabricated with rice straw and husk. Korean Journal of Chemical Engineering, 33(3), 851857. doi: 10.1007/s11814-015-0210-z

Zhu, J., Shi, Y., Fang, L., Liu, X., \& Ji, C. (2015). Patterns and determinants of wood physical and mechanical properties across major tree species in China. Science China Life Sciences, 58(6), 602-612. doi: 10.1007/s11427-015-4847-y
Manuscrito recibido el: 11 de octubre de 2016

Aceptado el: 13 de agosto de 2017

Publicado el: 23 de marzo de 2018

Este documento se debe citar como:

Musule, R., Bárcenas-Pazos, G. M., Pineda-López, M. R., Houbron, E. P., \& Sánchez-Velásquez, L. R. (2018). Desarrollo y evaluación de un método racional y no destructivo para la toma de muestras de maderas blandas utilizadas en análisis químicos. Madera y Bosques, 24(1), e2411427. doi: 10.21829/myb.2018.2411427

Madera y Bosques por Instituto de Ecología, A.C. se distribuye bajo una Licencia Creative Commons Atribución-NoComercialCompartirlgual 4.0 Internacional. 